\title{
Telemedicine Support in Zagreb
}

\author{
Major General Charles H. Roadmann II, MD \\ Deputy Surgeon General United States Air Force
}

\section{SLIDE 1:}

I believe we are in a time of historic convergence of historic factors. Everybody understands or feels the effects of the rate of change in all factors of our life. I believe that if we were able to look back 200 years, from the year 2200 , we would see this decade, or actually the last 100 years of the information age, as a time as disruptive as any major historic discontinuity. The current discontinuities are analogous to the fall of the Roman Empire, the dark ages, the renaissance, the invention of the Gutenberg press, the industrial revolution, the fall of the Russian Empire, and the advent of the information age. Consequently, most of us are uncomfortable. I think it's damn appropriate that we be uncomfortable, because we in fact, have two of these very rare historic discontinuities converging : the information age and the fall of the Russian Empire. We are witnessing the total restructuring of our postcold war economic environment with massive draw-downs, geo-political restructuring, and development of trigger technologies....the digital technology; which, I believe, will have social implications as far reaching as the invention of the Gutenberg press.

In health care the fundamental force that is shaping the industry is the transition from the traditional cottage industry organization to an integrated networked system. So, we ought to be pretty uncomfortable. Most of our previous speakers have made the point that this effort that we are addressing is not really about telemedicine, what we are talking about is a lot larger than that. It is, in fact, the reengineering of health care for the year $2020 \ldots$ or however long it's going to take to get the politics, technology, and organizations to retructure and resolve the national debate on healthcare. How we see the debate depends on our cohort. We look at this in a "Dickinsonian" sense as the "best of times" or the "worst of times". Most views seperate by cohort, education, tolerance for ambiguity and sometimes by imagination; but, clearly we have multiple groups that see what's going on today from entirely different perspectives and different degrees of agreement or alarm. 
What I believe is really important is that we must have a theory of how our system works and what I posit to you today is that we lack of a theory about how our system of healthcare works. In fact, for that reason most of the telemedicine stuff that began before ' 86 is now defunct. It was done under the theory, I believe, that it was good and interesting technology and therefore right ...but, this effort is not about black boxes and it is not about technology. It is about how we practice health care in totality and must be understood in an operational and exchange economy context.

\section{SLIDE 2:}

At the United States Military Academy they teach a concept called Threads of Continuity. They take young students and teach them why one field marshall is successful when another is not, when they meet in the same time space continuum....the battlefield. As the model is applied, there are multiple external factors to which each must react and a number of internal factors with which to react . External factors are shown on the outside of the triangle: economy, politics, doctrine, leadership, society, and technology -- those are things to which we must respond and that we do not control. We do not control the rate at which most of those things change. Yet, we have a job to do / product to deliver and we only have a strategy, tactics, logistics, administration, professionalism, and leadership, those factors inside the triangle, with which to posture our system to deal with those external factor and more importantly....the future!

I will give you a military example of how interrelated they are. During the Franco-Prussian War the French had a strategy of attack, attack, attack. The strategy worked very, very well, when troops could cover the ground between your trench and that of the enemy trench in a shorter time than the enemy could reload their musket. However, a technological event occurred, which should be considered a trigger technology, came through in the late 1800 's.....that was the machine gun. Regardless of technical change, the doctrine did not change. As a consequence, a generation of young men was lost in WW I. My point is that we must build an overarching theory about how our system might, or ought, work and identify the interrelated factors that drive change in the context of the environment...internal and external. 


\section{SLIDE 3:}

The situation we see today is of great technical innovation, disconnected efforts, and a resulting, what I would call a, dyssynergic non-systemic approach. That defines where we are today. However, there is a tremendous increase in a joint focus lead by Brigadier General Russ Zajtchuk, the telemedicine test bed, and OASD/HA. That effort and the fact that the Military Health Services System is that it is probably the only healthcare system large enough, or cohesive enough, to test the concepts of a unified theory of health care deliver into 2020 . Should really put the pressure on to design the system correctly..

\section{SLIDE 4:}

One of our most important goals is to have an over arching theory of telemedicine and its role in DOD. What we must do is: design it, understanding how it is today, understand how it ought to be and perform the gap analysis. The key focus must be interoperability and architectural control, making sure that we are plugging our capital investment into our larger problems rather than just the gadget $\underline{d e}$ jour. We must program an interoperable system with a strategy for peace and war.

\section{SLIDE 5:}

I would like to foot stomp one of the great dialogues that's going on. Telemedicine is being seen, I believe rightly so, as a great force multiplier in a war readiness combat environment. But, I would like to argue that it is also a great force multiplier in peacetime medicine. More importantly, if we do not implement it, get comfortable using it and incorporate it into the very process of delivering peacetime healthcare......we will also not use it in wartime either!

The peacetime approach has got to be in our TRICARE model of implementing virtual access, in the hub and spokeconfiguartion of MTF and clinics, with the cohorts of young practicing physicians using, depending on and comfortable with telemedicine to deliver efficient healthcare. The only difference between peacetime and wartime should be how rugged the equipment is. .. maybe the color of the box or how it packs upfor transportation... Not the practice. We have got to practice as we go to war. And I'm going to foot stomp that with Zagreb examples in just a little bit. 
The big advantage we will see in telemedicine is, in fact, taking us from the cottage industry organizational structure of healthcare to a large integrated system. That requires expanded situational awareness. I believe what we have now is a fundament lack of situational awareness of important issues in the delivery of our product such as:

-what kind of care did a casualty receive in the field treatment?

-what kind of care did a casualty in transportation?

-what kind of chronic problems does the patient have?

-what are the results of the test that was done?

I admit that I get angry every time I walk into the commissary. I see my can of beans go across the laser reader, I hear beep-beep and I know that the stock level has been adjusted, unit cost has been calculated and a re-order has been issued....that is real situational awareness at the point of service! We know all of the things about the system in the commissary, and we don't with our very important resources that we have in health care. We must develope a theory of healthcare... about how it ought to work and get on with the programming and implementation.

The issue in patient care is not just the cottage industry concept of what goes on across from the patient and the practitioner. The real issue is patient / practitioner relationship and the logistical support to make healthcare a seamless system. That is where telemedicine, I believe, will help us as we work the transportation, logistic support and the delivery of efficient / effective medicine to our patients.

\section{SLIDE 6;}

There is another clear issue, and that is what I prviously eluded to as the gadget de jour vs. the problems we need to solve. We need to have a balanced program between cutting edge technology and solutions to problems that are plaguing us day today. Technology solutions must fit into the current reality and the theory that we have of the future. It is clear to me that we need to get into a requirements pull rather than the technology push which we often see today. Now, I usually offend folks as I paraphrase the statement that war is too important to be left up to the General's and that is . telemedicine is too important to be left up to the technical folks....because what we get into is a 
technology push rather than a requirements pull. That inexorably leads us into the gadget de jour or what we can do vs. what we need to do from an operators perspective.

So, I think there is a mind set that we clearly have got to get into, and this mindset is going to decide whether we are either successful or not successful. ..it needs to focus around Commercial off the shelf (COTS) or Government off the shelf (GOTS) mature technology deployment and rapid technology deployment for efficiency and effectiveness of production, not just technical toys.

\section{SLIDE 7:}

Now, I'll talk about force projection. Because that is where the rubber really meets the road. The important issue for our system is to understand is that we run peacetime health care; but, the reason for military medicine is the support of combat arms. Medics are constantly in a "wind shear" because the combat CINCs need more "tooth to tail" in their force structure. We are in the process now of decreasing the theater footprint (hardware, manpower, equipment) which significantly changes the scope healthcare in the combat zone and transforms problems from a healthcare issue to a transportation issue. This is concept extremely important, and our emerging theory must be able to solve many of the problems by teledeployment.

\section{SLIDE 8:}

Deployment is not a theoretical construct in the post Desert Shield/ Storm environment. These are the places that we have medics deployed in an Ops tempo supporting the "New World Order".

\section{SIIIDE 9;}

The concept of operations that we are talking about is peacetime virtual access, wartime telemobilization, peacetime regionalization, wartime theater support, and in all of these conditions we are talking about the difference in Negreponte's terms of concentrating on bits versus the atoms. That is a cultural issue, a doctrinal issue and a practice of medicine issue.

\section{SLIDE 10;}

I want to talk about Zagreb: hardware, culture and utilization. 


\section{SLIDE 11:}

Hardware, I think, is the least interesting part of the telemedicine story. That's just a black box issue. Most of this stuff is technologically possible. This is what's deployed in Zagreb, Croatia.

\section{SLIDE 12:}

Utilization. Now you need to understand about Zagreb. The delivery platform is a Navy Fleet Hospital, with 60 inpatient beds, has been operated by the Army, Navy, and the Air Force on a rotating basis. So what I asked was, "give me the utilization." You can see the times that we were each there. Army was there from May '93 to '94. During that time 70 x-rays were transmitted and three VTC consultations. Navy was there from February '94 to '95. During that time they transmitted 9,700 images to San Diego and 9,900 images to Portsmouth. Air Force has been there since February '95. We have had 3 VTCs and they have primarily been around patient regulation. What I would emphasize on this slide, this is a cultural issue. If you don't use it in peacetime, you will not use it in wartime. The reason the Navy utilization is so high is that they are using an E-system shipboard teleradiology in daily practice. It is not foreign technology to them. Yet, everybody else is on a steep learning curve. So the balance between peacetime and wartime is a critical issue for effective use of the technology.

\section{SLIDE 13:}

And so our remaining task.... is moderately daunting. I believe our system is up to it, but we have got to develop an integrated theory. I believe the telemedicine test bed is going to do that for us. This needs to be a joint dream, a joint vision once the theory is there. The mission is just what we need to do. The strategy is just the scheme on how we are going to go do that. But it is clear to me that there is a lot of work that ahead of us.

Once we have the scheme, it is critical for telemedicine that DOD clearly build a CON-OPS, clearly develop metrics to tell us whether it is useful or whether it is not, and whether the deployment of telemedicine will increase the quality of healthcare and decrease the footprint while maintaing the capability to provide it. Up to this point, in Zagreb and every other deployment, it has subjectively increased the quality but has not decreased the footprint at all. 
And so, what we have got to do is validate our theory, deploy the technology, andand that's really a cohort cultural change.

Thank you. 


\section{NATIONAL FORUM: MILITARY TELEMEDICINE ON-LINE TODAY}

Major General Charles H. Roadman II, MD

Commander Air Force Medical Operations Agency

27-29 March 1995

\section{Vision Statement}

- Historic Convergence Forces

" Post Cold War

"Economic Restructure

» TriggerTechnologies

" Cottage Industry to Integrated System

- More than Telemedicine

- Reengineering Healthcare

- Window of Risk vs Opportunity 


\section{FORCES OF CHANGE}

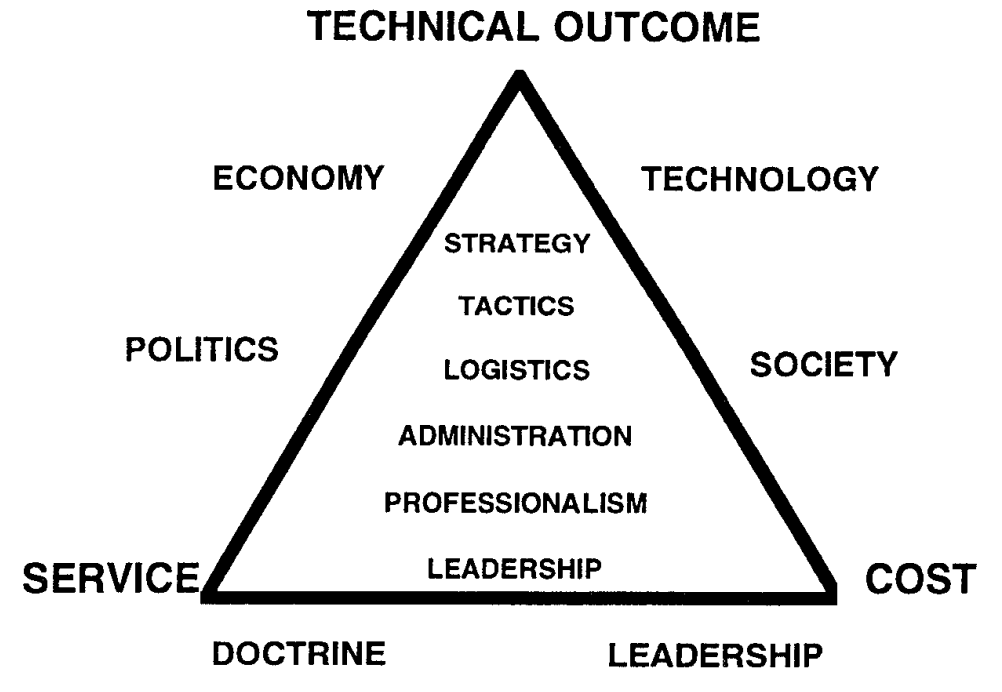

\section{Today's Situation}

- Great Technical Innovation

- Disconnected Efforts

- Resulting Dyssynergy

- Increasing Joint Focus

- Telemedicine Testbed 


\section{Goal and Objective}

- Theory of Telemedicine in DOD

- Design

»As Is

"Ought to be Vision

" Gap Analysis: Mission and Strategy

- Interoperability

- Programming of Strategy...Peace \& War

\section{Peace and War}

- TRICARE

- Support of Deployed Forces

- Situational Awareness..Reactive

Replanning
" Patient care
"In-Transit-Visibility
" Logistic Support
" Transportation 


\section{Research}

- System View Must Have Balance

"Near Term Issues

"Out Year "Cutting Edge"

- Requirement Pull vs Technology Push

- Business Case
" COTS / GOTS Focus
" Mature Technology Deployment
»Efficiency / Effectiveness....or just toys

\section{Force Projection}

- Support of Combat Arms

- Trade off Beans vs Bullets

»Strategic Lift....Cube and Weight

" Theater Footprint

" Theater Evacuation

» Tactical Integrity

- Teledeployment 
Where the Rubber Meets the Ramp

- TRICARE

- Military Operations (War and Other

Than War)

"Somalia

" Guantanamo Bay

» Haiti

»Panama

" Croatia

"Macedonia

\section{Concept of Operations}

- Peacetime

"Virtual Access

»Regionalization

» Bits vs Atoms
- Wartime

"Telemobilization

"Theater Support

"Bits vs Atoms 


\section{Focus on Zagreb}

- Hardware

- Culture

- Utilization

\section{Hardware}

- Components

" Digital Still Imagery (Kodak)

" VTC (CLI)

"Teleradiology (E-Systems)

- Communication Media

" Land Lines

"Satellite

- INMARSAT

- Bandwidth Limitations 


\section{Utilization}

- USA (May 93-Feb 94)

"70 Xrays

" 3 VTC Consultations

- USN (Feb 94-Feb 95)

"Zagreb to San Diego 9700 Images

"Zagreb to Portsmouth 9900 Images

" 9 AE Prevented

- USAF (Feb 95- Present)

" 3 VTC for Patient regulation

\section{Remaining Tasks}

- Integrated Theory

- Joint Vision....The Dream

- Mission.... What to Be Done

- Strategy....The Scheme

- Utilization Construct

¿CONOPS

"Metrics

"Validation of Theory

- CHANGE THE CULTURE 